\title{
Transformer of $\operatorname{tg} \delta$ on MSP430F1331 single chip microcomputer WANG Han $^{1}$ CAI Xinjing ${ }^{1}$,XiaoJieping ${ }^{2}$,Liu weiqing ${ }^{2}$ \\ ${ }^{1}$ School of electrical engineering Northeast Dianli University, Jilin,132012, Jilin China \\ ${ }^{2}$ School of electrical engineering Northeast Dianli University, Jilin,132012,Jilin China 2274556659@qq.com
}

Keywords: high voltage insulation, $\operatorname{tg} \delta, \operatorname{msp} 430 f 1331$

\begin{abstract}
This article designs a insulation dielectric loss detecting instrument based on MSP430F1331 single chip microcomputer, which is a research on $\operatorname{tg} \delta$ value detection of dielectric oil and insulating sleeve. The research shows the application of the new information collection terminal and new algorithm and TG delta detection is realized. It is turned out that the new insulation dielectric loss detecting instrument has the characteristics of low power consumption, high efficiency, accurately compared with the AI-6000E dielectric loss test instrument (the method of the resistant bridge.
\end{abstract}

\section{Introduction}

High pressure equipement plays an important role in power system's safe operation,which has far-reaching influence on power system. The problem of high pressure equipment will result in large area blockout accident, even the system paralysis in electric power system. Therefore, the maintenance of high pressure equipment plays an important part in routine maintenance. Dielectric loss test is an effective way to insulation defect detection in high voltage electrical equipment. Traditional dielectric loss test usually use dielectric detector based on schering bridge merhod to detect the loss of insulating medium for high voltage equipment such as transformer.But there are many shortcomings, such as: power consumption, poor anti-interference ability,complex operation an so on. These shortcomings are difficult to meet the demand of high efficiency, precision, low power consumption of data collection in modern power system. The design in this article uses a insulation dielectric loss detecting instrument based on MSP430F1331 single chip microcomputer. It's sampling section combines many kinds of integrated circuit. This dielectric loss meter improves the stability and accuracy of information sampling with low power consumption, fast operation, processing power and other advantages.

\section{Basic composition and working principle of the system}

Dielectric loss detector hardware system based on MSP430F1331 single chip microcomputer is divided into two parts: data acquisition and data processing. When the detector works, voltage sampling system firstly carries out voltage test to the detected object. Then current detection system samples the delay time of sampling to the object to be measured. $\triangle \mathrm{T}$ It' s controlled by the clock module in MSP430F1331 chip. Sampling system gets the sampling information of multiple sampling periods and sends the information to the data processing system. The data processing system combines ways of Pass Zero Distinguishing Phase and Least square value, processes the data repeatedly to get the tangent and sends to LED display system and storage system.

\section{System hardware circuit design}

\subsection{System logic diagram}

The overall block diagram of the hardware system is shown in Figure 1. The system is composed of two parts of the simulation board and 430 single-chip microcomputer. The simulation board includes sine wave sampling module, waveform processing module and waveform integrated module three parts. 430 single chip microcomputer is data processing module. 


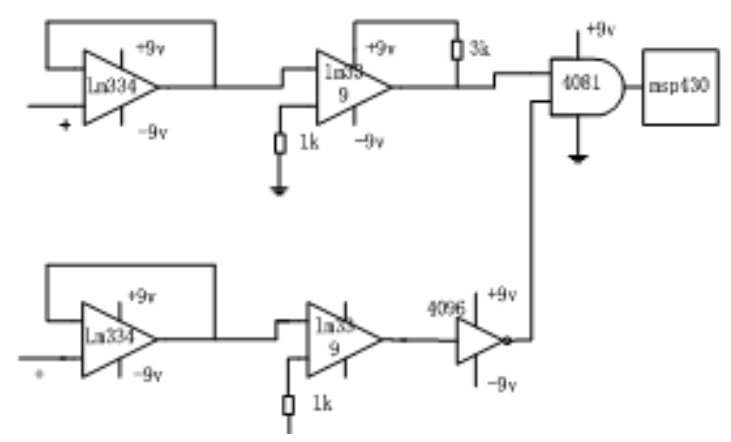

Fig.1 Hardware logic diagram

\section{2 waveform sampling module}

In order to ensure no distortion effect to the maximum degree of the waveform of the acquisition, LM334 is used as Voltage Follower to sample the waveform sampling. The advantages of voltage follower are very high input impedance and low output impedance, which are the most commonly used impedance transformation and matching circuit。Voltage follower is usually used as in buffer stage and out buffer stage of circuit. As high impedance input level of the whole circuit, it can ease the effect on the signal source; as low impedance output stage of the whole circuit, it can improve the ability to load. LM334 is the three terminal adjustable constant current source device. In the working current, the constant current source can be adjusted to 1000:1 and has a dynamic voltage range of $1-40 \mathrm{~V}$ wide, whose constant flow characteristic is very good, It is characterized by no need of precision voltage amplification, compensation, etc. To sum up, LM334 is used as the voltage follower to carry out the waveform sampling, The distortion of the waveform is reduced to the maximum extent.

\section{3 waveform treatment module}

Algorithm used in data processing module is high order fitting algorithm based on zero crossing time difference method. In the algorithm, the time delay difference is obtained and is transformed into a certain width $\triangle T$ square wave signal. The dielectric loss value of the sample can be obtained by measuring the width of the square wave. As a result, it is the most important factor in the acquisition of dielectric loss to obtain a square wave signal with good, no distortion, and a certain anti jamming ability.

This system uses LM339 to transform the sine wave as zero comparator Zero comparator's significance lies in Threshold Voltage UT $=0 \mathrm{~V}$ 。 When Integrated Operational Amplifier lies in open ring state, the output voltage is +UOM or -UOM. When the input voltage is $\mathrm{uI}<0 \mathrm{~V}$, $\mathrm{UO}=+\mathrm{UOM}$; When the input voltage is $\mathrm{uI}>0 \mathrm{~V}, \mathrm{UO}=-\mathrm{UOM}$ 。 The advantages of the net input voltage of integrated operational amplifier and net input current is approximately zero so as to protect the input level. Because the integrated operational amplifier does not work to the nonlinear region, at zero input voltage its internal transistor do not need to enter the saturated zone gradually from the end zone, or gradually from the saturated zone to the end zone.So it improves the output voltage. LM339 is an integrated circuit of four voltage comparator . The circuit has characters as follows: Operating power supplies voltage range ; both single power supply and dual power can work. Single power supply: $2 \sim 36 \mathrm{~V}$, Double power supply: $\pm 1 \sim \pm 18 \mathrm{~V}$; The current consumption is small, Icc $=1.3 \mathrm{~mA}$; Input offset voltage is small, $\mathrm{VIO}=+2 \mathrm{mV}$; Common mode input voltage range, Vic $=0 \sim$ Vcc-1.5V; Output compatible with TTL, DTL, MOS, CMOS, etc. The output can be connected with an open collector "or" door. The system uses LM339 as a zero comparator with low power consumption, protection of input stage, improve the speed of output waveform changes.

\subsection{Waveform integration module}

The signal delay difference of $\mathrm{T}$ square wave signal is the key to calculate the dielectric loss value.In the data processing module, the sine wave signal has been changed to square wave signal。 But at this time of the wave type signal is a two way slightly delayed square wave signal。To abtain delay signal difference, this system combines 4096 Not Gate and 4081and GAT in order to get 
$\triangle T$ 。Figure 2 is the schematic diagram of the wave. In Fig. 2 a), the waveform of the current input signal is adjusted by the LM339 of the square wave.Figure b) the waveform of a square wave of a voltage input signal by LM339. Figure C) as the input voltage signal through the 4096 NOT gate signal, Figure d) for current and voltage inversion after 4081 and the difference of the integration time delay signal waveform $\mathrm{T}$ 。 Figure 3 is the real acquisition of delta $\mathrm{T}$ waveform。

\section{Data processing}

Data processing applies to MSP430 series and F1331 as the core CPU data calculation 。 Measures of Pass Zero Distinguishing Phase and Least square value are taken to process $\mathrm{T}$ square wave signal . (Zero crossing method The current flowing through the I to measure the dielectric loss Voltage at both ends of the insulation U Frequency as a sine wave of $50 \mathrm{HZ}$ ) Through the measurement of sine wave current, voltage changed from negative to positive over time difference $\mathrm{T}$ zero count pulse in time domain and then converted into phase I ahead of $\mathrm{u}$ and TG delta phi difference。

$$
\begin{gathered}
\delta=(\pi / 2)-\psi=(\pi / 2)-2 \pi(\Delta T / T) \\
\Psi=2 \pi \quad(\Delta T / T)
\end{gathered}
$$

MSP430F1331 with high performance analog devices can be used for accurate measurement 。 Integrated 12 is A/D converter for 200ksps, coming with 16 bit RISC CPU and $125 \mathrm{~ns}$ instruction cycle. High performance applications can be achieved with less code space.And it has the high storage space in the single chip microcomputer $64 \mathrm{~KB}$ 。 In consideration, a high order algorithm is based on the zero crossing method of zero crossing. In data acquisition, efficient collection of high efficiency is done.Collection period is 0.2 seconds 100 times. In each collection, Zero crossing operation is used to find out the $\delta$ delta value .Single chip registers then store it. When it reached 1000 times, computation of least square values is used to ensure data accuracy and reliability. If we set $\sin \delta$ to $\mathrm{X}$, and set $\cos \delta$ to $\mathrm{y}$, the following functions can be obtained。

$$
X=\sum_{i=1}^{1000}\left[D_{0} \sin \omega t_{i}+D_{1} \cos \omega t_{i}-i\left(t_{i}\right)\right]^{2} \quad \text { (3) } Y=\sum_{i=1}^{1000}\left[C_{0} \sin \omega t_{i}+C_{1} \cos \omega t_{i}-u\left(t_{j}\right)\right]^{2}
$$

From the above 1000 operations can be linear equation

From the above 1000 operations can be linear equation

$$
\begin{gathered}
A=\left[\begin{array}{ll}
\sin \omega t_{1} & \cos t_{1} \\
\sin \omega t_{2} & \cos t_{2} \\
\ldots & \\
\sin \omega t_{1000} & \cos t_{1000}
\end{array}\right], \quad G=\left[\begin{array}{c}
i\left(t_{1}\right) \\
i\left(t_{2}\right) \\
\ldots \\
i\left(t_{100}\right)
\end{array}\right], \\
F=\left[\begin{array}{c}
u\left(t_{1}\right) \\
u\left(t_{2}\right) \\
\ldots \\
u\left(t_{1000}\right)
\end{array}\right], \quad D=\left[\begin{array}{l}
D_{0} \\
D_{1}
\end{array}\right], \quad C=\left[\begin{array}{c}
C_{0} \\
C_{1}
\end{array}\right]
\end{gathered}
$$

$\delta_{\text {平 }}=\pi / 2-\psi$ Solving the linear equations , can get $\mathrm{D}_{0} \mathrm{D}_{1} \mathrm{C}_{0} \mathrm{C}_{1} \quad \psi=\psi_{\mathrm{i}}-\psi_{u}$, and then get, Through $\delta_{\text {平 }}$, we can get the most accurate dielectric loss value 


\section{Experimental results are compared with the AI-6000E dielectric loss test instrument (the method of the resistant bridge).}

This experiment is done in the field of high voltage electric laboratory of Northeast Dianli University.Samples were collected for SFZ7-50000/110, transformer insulation medium oil and B C A three insulation casing. And the influence of the electric field intensity on the data is considered. Specific data are shown in Table 1 and 2:

Tab.1Transformer insulating oil test

\begin{tabular}{|c|c|c|c|}
\hline \multicolumn{2}{|c|}{$\begin{array}{l}\text { Field strength and } \\
\text { influence of experiment }\end{array}$} & $\mathrm{AI}-6000 \mathrm{Etg} \delta \%$ & $430 \operatorname{tg} \delta \%$ \\
\hline 1 & Nofieldstrength & 0.141 & 0.141 \\
\hline \multirow[t]{2}{*}{2} & 50000/110Trans & 0.146 & 0.141 \\
\hline & \multicolumn{3}{|l|}{ former } \\
\hline 3 & $110 \mathrm{kV}$ & \multicolumn{2}{|r|}{$0.141-0.1413$} \\
\hline \multicolumn{4}{|c|}{ Tab.2 Transformer bushing test } \\
\hline \multicolumn{2}{|c|}{ Measurement position } & $00 \operatorname{Etg} \delta \%$ & $430 \operatorname{tg} \delta \%$ \\
\hline & A & 0.439 & 0.433 \\
\hline & B & 0.328 & 0.325 \\
\hline & $\mathrm{C}$ & 0.355 & 0.355 \\
\hline
\end{tabular}

In Table 1, the different working conditions are selected to compare with the experimental results. The influence of high pressure on the electric field intensity in different working conditions is considered.The interference of the $110 \mathrm{kV}$ analog line is the most. The error of the AI- $6000 \mathrm{E}$ dielectric loss tester is $0.004 \%$. The error of the new type dielectric loss meter is $0.001 \%$. Electric field beside the lightning experiment machine is most unstable.Two dielectric loss testing apparatus are all interference。To sum up, new dielectric loss meter can work in different electric field strength, whose error is between $0.001 \%-0.003 \%$. In Table 2 , the position of the transformer insulation sleeve is fixed. The impact of other environmental factors can not be considered.The experimental comparison of two kinds of dielectric loss meter is carried out. From table 2 we can see that the experimental error between the AI-6000E dielectric loss tester $\operatorname{tg} \delta \%$ and $430 \operatorname{tg} \delta \%$ is between $0.003 \%-0.006 \%$. The accuracy of the 430 dielectric loss meter is demonstrated.During the experiment, the collection and operation time were measured. 430 dielectric loss meter is due to no need to adjust the capacitance resistance, which in the time greatly exceeds the AI-6000E dielectric loss tester.

\section{Conclusion}

1) The new integrated circuit information acquisition terminal is different from the traditional pulse sampling, which is easily influenced by electric field, temperature, etc, So it can improve the stability of the collection information.

2) Because of high performance of single chip microcomputer, the method of calculating the zero crossing time difference method and the least square method is used, which improves the accuracy of the data. Besides the calculation speed is improved compared with the traditional method of 
measuring instrument of the resistant bridge.

3 ) The whole hardware part uses 3.3V button battery power supply compared with the traditional dielectric loss meter can greatly save energy and reduce power consumption.

\section{References}

[1] Wu Weihan, Ma Weimin. Research on portable digital dielectric loss measuring instrument [J], high voltage technology, $1996.22 .1: 91-94$

[2] Gu Huixing. The design and implementation of the signal source design and Realization of the fine stability of the [D], Xi'an University of Science And Technology,2013

[3]Chen Haibing. Design of [J]. based on the MSP430F6736 of the full SOC single-phase smart meter,2015,04:8-12.

[4] Qiu Hong, a portable wireless multi-channel data acquisition system based on MSP 430 MCU (), the Journal of science and Science 2011,39,11

[5] Chen Yirong.LM334's special application [R] instrumentation technology and sensor,2006.12.no12

[6] Ma Weimin, Wu Weihan. Influence of power supply harmonics on dielectric loss measurement [J]. Journal of Tsinghua University (NATURAL SCIENCE EDITION),1997,37 ( 1 ):65- 68.

[7] Xie Nan, Chen Weimin. Design of grid connected inverter based on distributed new energy generation [J],2012,02:203-206.

[8]Yi Zhenhua, Sun Yize, Peng Lele, Chen Xiao. Power supply design of photovoltaic grid connected inverter [J],2012,08:214-217.

[9]Pang Qichang, Zhang Haifeng, Chen Xiuchun. Characteristics and detection of high voltage corona discharge $[\mathrm{J}]$. Electrical measurement and instrumentation,2006,02:6-8+31.

[10]Zhang Jing, Zeng Xianyun. Introduction of the embedded system [J]. Electrical measurement and instrumentation,2002,04:42-44.11. 